\title{
Berichtigung zu
}

\section{Zum Darstellungssatz fuir Siegelsche Modulformen}

Math. Zeitschr. 102, 30-43 (1967)

\section{Helmut Klingen}

Auf Grund verschiedener Anfragen bezüglich des Beweises von Satz 2 der oben genannten Arbeit möchte ich die folgenden Ausführungen machen, welche eine zumindest unvollständige frühere Argumentation berichtigen. Es handelt sich um den Nachweis von

$$
\Phi E_{n, r}^{k}(*, f)=\left\{\begin{array}{lll}
E_{n-1, r}^{k}(*, f) & \text { für } & 0 \leqq r<n \\
0 & \text { für } \quad r=n
\end{array}\right.
$$

und gerades $k>n+r+1$. Diese Aussage ist in Formel (12) der Arbeit enthalten bzw. trivial in den Fällen $n=1$ oder $r=n$. Es sei nun $n>1$ und $r<n$. Wegen der gleichmäßigen Konvergenz der Eisensteinreihen in Vertikalstreifen kann der $\Phi$-Operator gliedweise angewendet werden. Ist $M \in \mathfrak{C}_{n, n-1}$, so hängt das allgemeine Reihenglied für $Z=\left(\begin{array}{cc}Z_{1} & 0 \\ 0 & i \lambda\end{array}\right)$ nicht von $\lambda$ ab. Die Gesamtheit dieser Glieder ergibt

$$
\sum_{M:\left.\mathfrak{C}_{n, r} \cap \mathfrak{C}_{n, n-1}\right|^{\mathfrak{C}_{n, n-1}}} f\left(M\langle Z\rangle^{*}\right) M\{Z\}^{-k}=E_{n-1, r}^{k}\left(Z_{1}, f\right) .
$$

Es bleibt zu zeigen, daß die restlichen Reihenglieder der Eisensteinreihe von dem $\Phi$-Operator annulliert werden. Unter Verwendung der Majorante $G_{n, r}^{k}$ genügt hierzu der Nachweis von

$$
\lim _{\lambda \rightarrow \infty} \operatorname{det}\left(\operatorname{Img} M\langle Z\rangle^{*}\right)|M\{Z\}|^{2}=\infty, \quad Z=\left(\begin{array}{cc}
Z_{1} & 0 \\
0 & i \lambda
\end{array}\right)
$$

für jedes $M \in \Gamma_{n}$ mit $\mathfrak{C}_{n, r} M \cap \mathfrak{C}_{n, n-1}=\emptyset$. Setzt $\operatorname{man} Z_{1}=X_{1}+i Y_{1}$,

$$
C=\left(\begin{array}{cc}
* & * \\
C_{1} & \mathfrak{c}
\end{array}\right), \quad D=\left(\begin{array}{cc}
* & * \\
D_{1} & \mathfrak{d}
\end{array}\right), \quad H=Y_{1}\left[C_{1}^{\prime}\right]+Y_{1}^{-1}\left[X_{1} C_{1}^{\prime}+D_{1}^{\prime}\right]
$$

mit Matrizen $C_{1}, D_{1}$ von $n-r$ Zeilen und $n-1$ Spalten, so wird explizit

$$
\operatorname{det}\left(\operatorname{Img} M\langle Z\rangle^{*}\right)|M\{Z\}|^{2}=\lambda \operatorname{det}\left(Y_{1}\right) \operatorname{det}\left(H+\lambda c c^{\prime}+\lambda^{-1} \mathbf{b} \mathbf{b}^{\prime}\right) \text {. }
$$

Da Rang $\mathrm{D} \mathrm{D}^{\prime} \leqq 1$ ist, steht rechts ein Polynom in $\lambda$. Die obige Grenzwertrelation ist also wahr, sofern dieses Polynom positiven Grad besitzt. Angenommen sein Grad sei Null; dann ist sein Wert eine positive reelle Konstante gemäß 
der Bedeutung der linken Seite der letzten Gleichung. Die Abschätzung

$$
\lambda \operatorname{det}\left(Y_{1}\right) \operatorname{det}\left(H+\lambda \boldsymbol{c} \boldsymbol{c}^{\prime}+\lambda^{-1} \mathrm{D} \mathrm{D}^{\prime}\right) \geqq \lambda \operatorname{det}\left(Y_{1}\right) \operatorname{det}(H)
$$

zeigt zunächst $\operatorname{det}(H)=0$. Folglich ist $\left(C_{1} D_{1}\right)$ nicht von Maximalrang. Indem man also von links mit einer geeigneten unimodularen Matrix multipliziert, d.h. indem man über $M$ in seiner Restklasse modulo $\mathfrak{C}_{n, r}$ geeignet verfügt, kann man annehmen, daß die letzte Zeile von $\left(C_{1} D_{1}\right)$ verschwindet. Bezeichnet man noch mit $c$ bzw. $d$ das letzte Element der Spalte $c$ bzw. $\downarrow$, so ergibt sich unter Verwendung der Symmetrie von $C D^{\prime}$

Wäre $c \neq 0$, so würde

$$
d \mathfrak{c}=c \mathfrak{b} \text {. }
$$

$$
\operatorname{det}\left(\operatorname{Img} M\langle Z\rangle^{*}\right)|M\{Z\}|^{2}=\lambda \operatorname{det}\left(Y_{1}\right) \operatorname{det}\left(H+\left(\lambda+\frac{1}{\lambda}\left(\frac{d}{c}\right)^{2}\right) \mathfrak{c} c^{\prime}\right)
$$

folgen, und dieses Polynom in $\lambda$ hätte die Nullstelle $i d c^{-1}$ im Widerspruch zu der früheren Feststellung. Somit ist auch $c=0$, das heißt $\mathbb{C}_{n, r} M \cap \mathfrak{C}_{n, n-1} \neq \emptyset$. 